\title{
PECULIARIDADES DE LA ENSEÑANZA DEL TROMBÓN EN LA REALIDAD SOCIOCULTURAL DE LAS UNIVERSIDADES DE COLOMBIA
}

PARTICULARITIES OF TROMBONE TEACHING IN THE SOCIOCULTURAL REALITY OF COLOMBIAN UNIVERSITIES
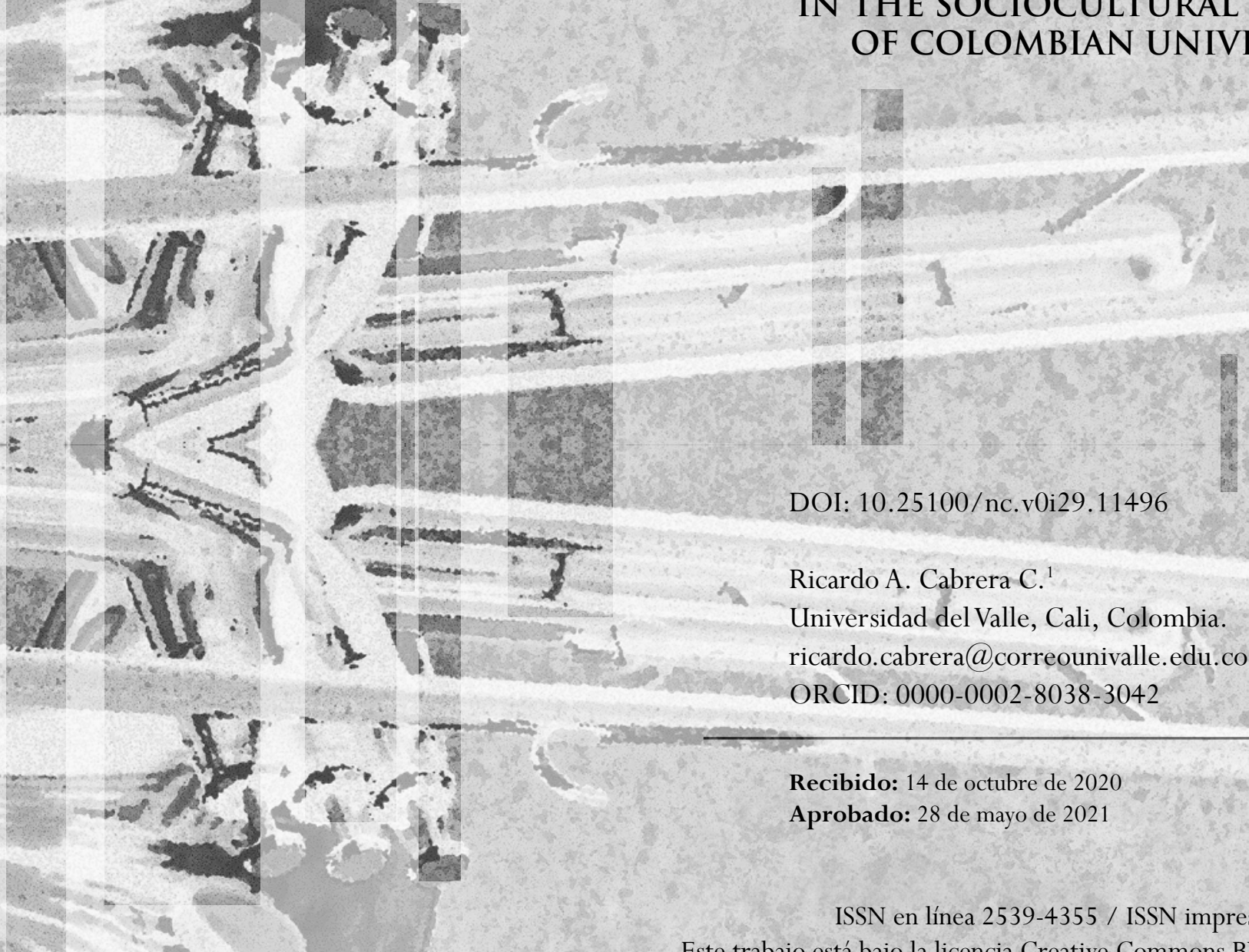

DOI: $10.25100 /$ nc.v0i29.11496

Ricardo A. Cabrera C. ${ }^{1}$
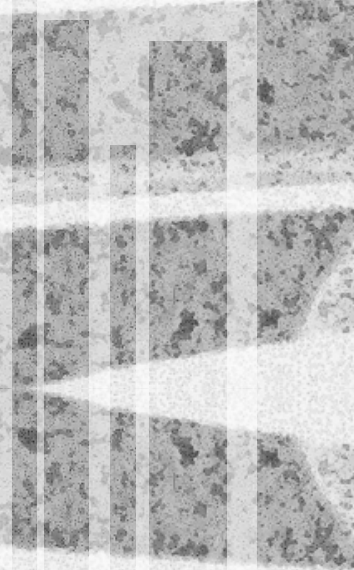
Universidad del Valle, Cali, Colombia. ricardo.cabrera@correounivalle.edu.co ORCID: 0000-0002-8038-3042

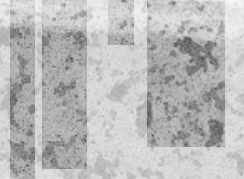

Recibido: 14 de octubre de 2020
Aprobado: 28 de mayo de 2021

ISSN en línea 2539-4355 / ISSN impreso 1900-9909 Este trabajo está bajo la licencia Creative Commons BY NC SA 4.0.

¿Cómo citar este artículo? / How to quote this article?

Cabrera, C. (2021). Peculiaridades de la enseñanza del trombón en la realidad sociocultural de las universidades de Colombia.l. Nexus, (29), 1-19. https://doi.org/10.25100/nc.v0i29.11496 
Resumen: En el marco de mi tesis doctoral Metodologías europeas y norteamericanas para la enseñanza de trombón en universidades públicas (1980-2016): adaptación a la realidad cultural y académica de Colombia he escrito un artículo que muestra las vicisitudes y dificultades de la inclusión de este pregrado específico, para ser entendido y asimilado, en las condiciones del andamiaje de las estructuras académicas, organizativas y conceptuales de la universidad pública. Esta, que asumió una carrera típica de la formación en conservatorio, no estaba preparada para esta inserción y muestra incomprensión con las características concretas del arte musical instrumental y su formación. Proponemos, a través de una investigación y análisis crítico, mostrar procesos necesarios de transformación en su implementación para que esta carrera tenga una inclusión equitativa, proficiente y esté acorde con las exigencias mundiales de competencia y calidad formativa.

Palabras clave: Trombón, Pedagogía del trombón, Métodos, Universidad Pública, Currículo, Instrumentista/educador.

Abstract: In the framework of my doctoral thesis European and north american methodologies for the teaching of trombone in public universities (1980-2016): an adaptation to the cultural and academic reality of Colombia', I have written an article that shows the vicissitudes and difficulties of the inclusion of this specific undergraduate degree, to be understood and assimilated, in the conditions of the structuring staging of the public university. This, that took on a typical career of 'training in a conservatoire', was not prepared for this insertion, and shows incomprehension of the concrete characteristics of the instrumental musical art and its training. We try, through research and critical analysis, to show the necessary transformation processes in its implementation, so that this career can have an equal and proficient inclusion and can be in accordance with the global demands of competence and training quality.

Keywords: Trombone, Trombone pedagogy, Methods, Public University, Curriculum, Instrumentalist/educator.

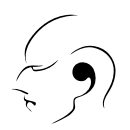

\section{Origen del artículo}

Este artículo de investigación hace parte de la tesis del autor en el contexto del Doctorado en Historia y Artes de la Universidad de Granada (España). Hace parte del Convenio Específico de Colaboración de la Universidad del Valle (Colombia), la Universidad de Granada (España) y la Asociación Universitaria Iberoamericana de Postgrado (AUIP) para la Puesta en Marcha de un Programa para Formar Doctores en Disciplinas Relacionadas con las Artes. 

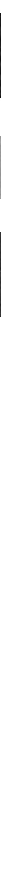

Conforme fue madurando la profesión, la música instrumental fue añadida a los programas escolares de música. Aquí nuevamente, existía un objetivo principal: enseñar a los jóvenes a tocar instrumentos musicales. El principio operativo era que la ejecución, y el contacto con la música, desarrollaba una comprensión musical, pero el aprendizaje se evaluaba en términos del comportamiento en la ejecución, más que en términos de entendimiento.

(Regelsky, 1980, p. 17)

La diversidad cultural que tiene nuestro país se refleja sin duda en las condiciones contextuales que afectan la consolidación de los programas creados por las unidades académicas de formación musical en los últimos años, y que implementan la relativamente reciente carrera de Trombón como instrumento principal. No obstante, y a pesar de existir de vieja data su enseñanza en los conservatorios, solo en los últimos años del siglo XX y en los comienzos de este, se consolida un programa específico de instrumento (pregrado de trombón) instituido en sus aspectos y regulaciones competentes y legales en la universidad pública.

Por lo encontrado en las universidades visitadas en el marco de esta investigación (Nacional, del Valle, de Antioquía, Cauca, Tecnológica de Pereira, Caldas) salta a la vista que el modo de ir generando un pensum para el instrumento, y consecuentemente unas metodologías, ha recorrido mil vicisitudes para su afianzamiento curricular en las propias escuelas o departamentos de música ${ }^{2}$. Numerosos prejuicios, posturas conceptuales, la propia novedad de la implementación de un programa para instrumento hicieron que este fuera desligado de dos realidades: el alumno y su contexto, y el instrumentista profesor. Las escuelas o departamentos de música trajeron esta "nueva materia" usando sus propios procedimientos de implementación pedagógica aplicados en otras áreas de la carrera musical; el alumno de instrumento (de música como una generalización pertinente) entraría al mundo formal educativo universitario con sus códigos, y él, y su maestro, deberían simplemente encajar. Dos factores eran desconsiderados: el social-cultural del estudiante y la capacidad pedagógica del instrumentista-profesor. Cárdenas Soler refiriéndose al tema señala: 
Dussel (2006) y Díaz \& Inclán (2001) explican cómo Latinoamérica, a partir de 1990, impulsó el crecimiento y desarrollo de su sistema educativo, caracterizado hasta entonces y aún hoy por una cultura política que fomentó la inequidad e ineficiencia, promoviendo la inclusión, la cobertura, el cambio curricular y la transformación de las prácticas escolares. Pese a las buenas intenciones, algunos problemas siguen subsistiendo: las precarias condiciones de trabajo y recursos de las escuelas, el limitado apoyo de la comunidad y del gobierno, la necesidad de persuasión para la matrícula de estudiantes y, sobre todo, la desfavorable formación del profesorado y posicionamiento de la carrera docente (Dussel, 2006; Díaz \& Inclán 2001, citados en Soler, 2012, p. 196).

Estos factores siguen siendo decididamente vigentes, y pese a que muchas investigaciones han dilucidado alternativas y soluciones, estas continúan siendo letra muerta, referencias bibliográficas y elucubraciones teóricas, entre otras. Lamentablemente poco o nada se las tiene en cuenta, a pesar del supuesto de que para eso se desarrolla la investigación en la universidad. Los problemas sociales y pedagógicos descritos en tales documentos investigativos aún tienen vigencia, en mayor o menor grado, en nuestras instituciones musicales públicas.

Una primera reflexión importante, para observar la ausencia de políticas académicas claras y objetivos específicos, es la que se refiere a la comprensión, estudio y misión con la que se establece el perfil de la población a donde está direccionada. El promedio de aspirantes con deseo de formarse en trombón en una universidad parte de factores diferentes al promedio de otras carreras. Las fuentes "inspiradoras" de esta aspiración no son necesariamente la vida escolar de los jóvenes. Por lo regular las motivaciones están ligadas a su participación en bandas de vientos de las poblaciones (pueblos como coloquialmente se les llama), sobre todo en el centro de Colombia y la región cafetera y antioqueña. En los Departamentos de Cauca y Nariño, lugares del sur del país con tradición también de bandas, igualmente estas son alentadoras de tal acometido; en el Valle de Cauca existe un fenómeno popular-comercial muy particular que genera una vinculación amateur temprana al instrumento y el deseo de ser trombonista para tocar en una banda de música: la así llamada "Salsa". Ulloa (1989) dice:

En Cali este fenómeno artístico urbano, como lo ha sido el tango en Buenos Aires, ha contagiado y estimulado a la juventud como un "destino" de vida. La teatralidad de sus historias y personajes, el barrio como el lugar de realización del sueño, de convertirse en figura de su entorno, representa un factor definitivo en el deseo de los jóvenes de transformarse en los "héroes" populares de este género musical (p.16).

Sin duda tal proliferación de grupos se transforma en un mercado para profesionales del trombón. 
En menor cuantía otros interesados provienen de experiencias escolares (como se presumiría debería ser), y, que como en otras áreas de conocimiento, hayan sido estimulados en la clase de música en su escuela o colegio, dado el carácter formador de conocimiento y realización de talento artístico de esta materia. Existe una voluntad social en sectores de la clase media por "educar a sus hijos en arte" (Revista Semana, 2020), que no responde a políticas educativas estimuladas y postuladas desde el Estado: son actos de autodeterminación social basados en tradiciones pequeño burguesas heredadas de costumbres arraigadas en el ADN histórico. Hoy en día, los cambios abruptos que la globalización y el capitalismo salvaje trajeron a la familia, transformaron la interacción de sus miembros en todos sus ámbitos; los hogares en la actualidad son obligados a ocupar a los hijos todo el día en "algo" (como clases de música, por ejemplo) para poder cumplir con los tiempos que el trabajo roba a los padres para interactuar en los procesos de la vida formativa de sus hijos.

Como si esto fuera poco, el gobierno de Colombia ha hecho gran y estruendosa apología conceptual sobre las así llamadas “competencias artísticas”, apoyándose en el concepto "campo" de Pierre Bourdieu (2005), para justificar, con "argumentaciones doctas” (p. 96), la importancia de descalificar la enseñanza específica de la música en las escuelas y colegios; esto replantea la pertinencia y existencia actual de las licenciaturas específicas en artes: música, artes plásticas, teatro, danza, etc. (Ministerio de Educación Nacional, 2010, pp. 14-17). Asimismo, en algunas universidades la educación musical para niños y jóvenes es manejada a través de la idea, poco plausible, de generación de recursos propios con “cursos de extensión”, como respuesta frente a la desprotección presupuestal del Estado y la propia institución. Es un "negocio" (que no da recursos realmente) y no una actividad formadora preuniversitaria en instrumento-música realmente. En el caso de ciertas universidades encontramos que los docentes son egresado recientes o alumnos de último año, no hay planes ni congruencia programática preparatoria para el ingreso al pregrado.

Por tanto, el interesado en ingresar a una carrera instrumental se enfrenta a muchos retos en términos de su formación educativa en general y musical e instrumental en particular. Este fenómeno ha traído consigo la dificultad de la heterogeneidad formativa y social de la población a recibir en los programas de música-instrumento. Aunque este inconveniente fue entendido formalmente en muchas instituciones, esto es, era necesario crear un programa pedagógico profesional en los niños (como fue en los conservatorios), existen diferencias en los programas de las universidades para establecer parámetros similares que garanticen un complemento didáctico idóneo en este segmento poblacional infantil. Algunas universidades muestran progreso y dinamismo y otras aún no. 
Por otro lado, está la formulación de los sistemas de organización curricular en general y del pensum en particular. Los modelos autónomos de estas regulaciones, que cada universidad crea para su implementación, no necesariamente son funcionales para los programas de especialización instrumental, parten de sistemas generalizados en la universidad, o de sistemas que funcionan en áreas de la música diferentes y que no se corresponden con el sentido de la educación instrumental. Reafirmamos: la carrera de instrumento es un pregrado específico.

Por tanto, el sistema universitario debe rehacer y reformular este aspecto definitivo para poder desarrollar una carrera de pregrado idónea en trombón. Es necesario que los postulados formales transciendan y sean relevantes para mejorar el estudio de las singularidades de la población aspirante en todos los aspectos, esto es...

...Un proceso que exige estudiar y explorar esas nuevas realidades sociales, culturales y funcionales de los estudiantes y revisar los perfiles de ingreso y egreso conforme a los criterios y lineamientos de la nueva política curricular, para intervenir de la mejor manera el Proyecto Formativos de la Universidad (Acuerdo 025 2015, Universidad del Valle, p. 32.)

Vemos como en la práctica es común diseñar textos programáticos que no pasan del papel, que han caído en la repetición formal y mecánica, en discusiones vagas, pero que no son aplicados ni utilizados como procedimientos de partida para la formulación y aplicación de políticas que permitan comprobar su sapiencia, practicidad y vigencia en el objeto social: los estudiantes. Las unidades académicas se preocupan solamente por llenar las vacantes de cada nuevo período de admisiones, el discernimiento ideológico que está planteado en la visión y misión de las universidades no hace mella conceptual, y no se generan preguntas importantes del para qué, el porqué, hacia dónde, en qué condiciones sociales, cuál es el papel de nuestra carrera musical con la sociedad, al recibir a sus jóvenes para darles la oportunidad de acceder a un diploma profesional en instrumento. El formulismo no debe reemplazar la acepción "educación formal".

Dentro de las incongruencias están también los recursos: es notorio cómo salones especiales, salas, instrumentos (algunos muy costosos), etc., no han tenido preeminencia en las universidades públicas; no hay salas de conciertos, la mayoría han adaptado auditorios como tales; no existen líneas de crédito y apoyo a los alumnos para la compra de instrumentos, los sistemas de seguros para estos son absurdamente caros y en muchos casos las aseguradoras no los cobijan. Las universidades públicas representan la única opción para la población de escasos ingresos, y deberían ser las promotoras del eufemismo del artículo 67 (constitución de Colombia Artículo 67).

La universidad pública de Colombia adolece de implementación eficiente y de última generación de nuevas tecnologías. Las diferencias entre universidades públicas por regiones en este aspecto son vastas y evidentes. 
...daba al estudiante cierta idea de su cierta posición en un curso determinado. Para proporcionar una medida uniforme de la cantidad de tiempo dedicada a un propósito determinado, la hora-crédito o unidad fue aceptada ampliamente (Panquevo, 1980, p. 12).

Este sistema perduró inamovible durante muchos años y, como suele suceder, terminó por perder la eficiencia y sapiencia que generó sus orígenes. Poco a poco se fueron dilucidando aspectos, que, para nuestro entender, deberían ser repensados en el caso de la educación artística instrumental. Se convirtió el sistema de créditos en una barrera formal y burocrática que impide equidad y holgura a la carrera de instrumentista. Además, en nuestro país hay una tendencia tácita y generalizada a adoptar mecanismos foráneos a imagen y semejanza de cómo fueron aplicados y usados en otro tiempo y lugar, al mismo tiempo con retraso para instalarlos en nuestro medio y sin mediar ningún rigor sobre sus particularidades. Este hábito se cuela en la reglamentación que los organismos encargados de las políticas y directrices educativas del Estado apropian y establecen como ley para el uso en la educación pública, en 1980, por ejemplo, el artículo 40 de la Ley de Educación decía:

Los programas de las diversas modalidades educativas del Sistema de Educación Superior se organizarán con base en unidades de labor académica, cuya definición tendrá en cuenta un valor para la actividad teórica y otro para la práctica (citado por Panquevo, 1980, p. 1).

Esta disposición fue derogada por la ley 30 de educación de 1993, pero mantuvo su esencia en cláusulas sobre valores del crédito académico y su fórmula de 48/16. Sin embargo, se añadió a posteriori una reglamentación a la ley referente a lo que se denominó "Registro Calificado", que se estableció como condición para que el ministerio "otorgue" este mérito a las instituciones universitarias que cumplan con una serie de requisitos establecidos en ella; esta reglamentación fue instituida en el Sistema de Aseguramiento de la Calidad de la Educación Superior, Capítulo 2 y 7 del Título 3 de la Parte 5 del Libro 2 del Decreto 1075 de 2015 -Único Reglamentario del Sector Educación. Allí encontramos el entendimiento gubernamental sobre los créditos y su rol en la acreditación:

De la Evaluación para el Registro Calificado

Artículo 2.5.3.2.3.2.1. Sobre los Créditos Académicos.

Las instituciones de educación superior definirán la organización de las actividades académicas de manera autónoma. Para efectos de facilitar la movilidad nacional e internacional de los estudiantes y egresados y la flexibilidad curricular entre otros aspectos, tales actividades deben expresarse también en créditos académicos.

Los créditos académicos son la unidad de medida del trabajo académico para expresar todas las actividades que hacen parte del plan de estudios que deben cumplir los estudiantes, sin exceptuar monografías, trabajos de grado, tesis o sus equivalentes en el caso en el que proceda. 


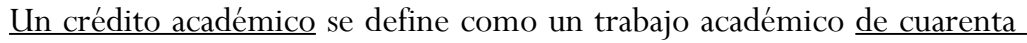
y ocho (48) horas que comprende las horas con acompañamiento directo del docente y las horas de trabajo independiente que el estudiante debe dedicar a la realización de actividades de estudio, prácticas u otras que sean necesarias para alcanzar las metas de aprendizaje.

Artículo 2.5.3.2.3.2.2. Número de créditos de la actividad académica. El número de créditos de una actividad académica en el plan de estudios será aquel que resulte de dividir en cuarenta y ocho (48) el número total de horas que debe emplear el estudiante para cumplir satisfactoriamente las metas de aprendizaje.

Artículo 2.5.3.2.3.2.3. Horas con acompañamiento e independientes de trabajo. De acuerdo con la metodología del programa y conforme al nivel de formación, las instituciones de educación superior deben discriminar las horas de trabajo independiente y las de acompañamiento directo del docente.

Para los efectos de este Capítulo, el número de créditos de una actividad académica será expresado siempre en números enteros, teniendo en cuenta que una (1) hora con acompañamiento directo de docente supone dos (2) horas adicionales de trabajo independiente en programas de pregrado y de especialización, y tres (3) en programas de maestría.

Lo anterior no impide que las instituciones de educación superior puedan proponer el empleo de una proporción mayor o menor de horas con acompañamiento directo frente a las independientes de acuerdo con la naturaleza del programa y su nivel de formación.

En los doctorados, la proporción de horas independientes podrá variar de acuerdo con la naturaleza propia de este nivel de formación (Decreto 1075 de 2015) $)^{3}$.

Como se aprecia, la esencia matemática de la cuenta se mantuvo, pero aparecen opciones interesantes como la que dice: "lo anterior no impide que las instituciones de educación superior puedan proponer el empleo de una proporción mayor o menor de horas" (Decreto 1075 de 2015), lamentablemente esta flexibilidad propuesta no alcanza a tener pináculo en cuanto la clase de instrumento como pregrado específico.

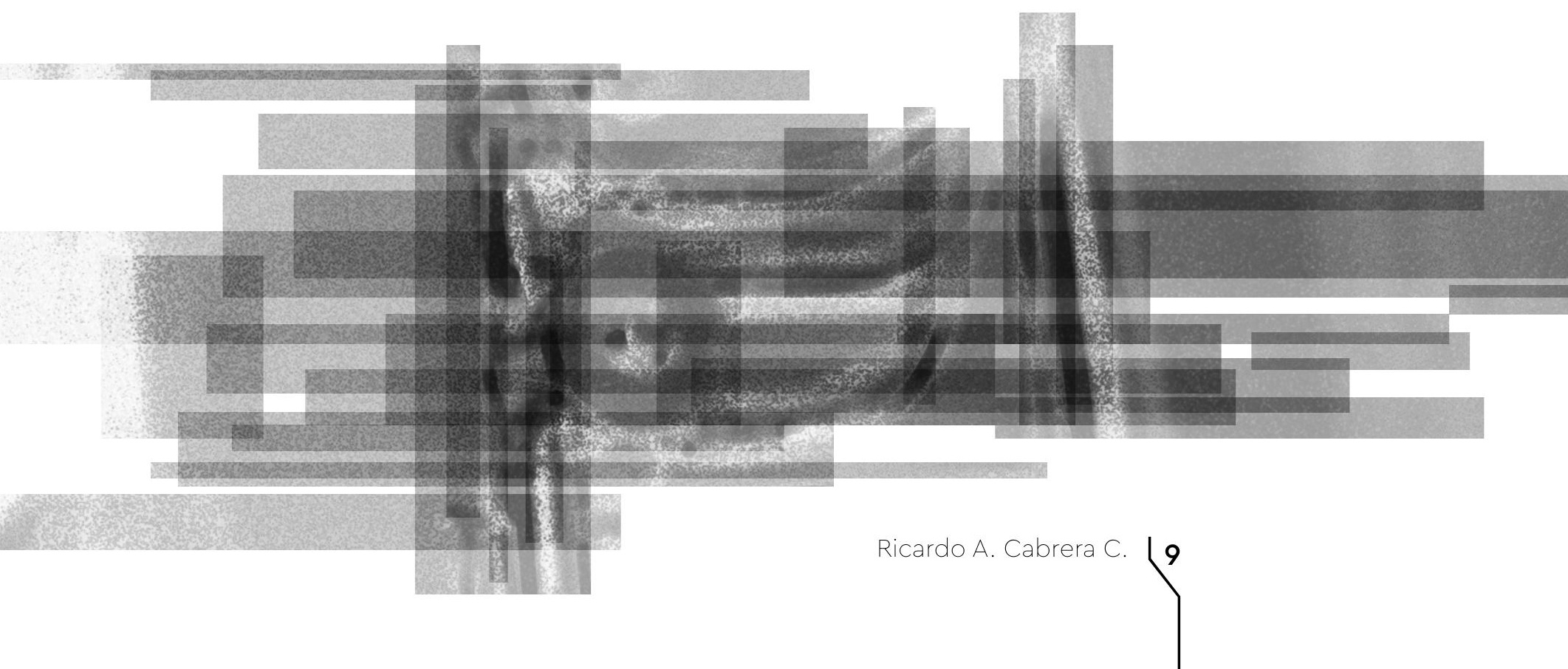


Con los créditos se generó una camisa de fuerza para usar en la subasta de contenidos del pensum de una carrera, en el cual todos los interesados ofrecen sus arguyas como el mejor postor. Estos se trasformaron en la "solución" a los problemas de la calidad académica al desarrollar e incrustarlos en currículos presumiblemente "transformadores", pensando que el cambio de contenidos curriculares, y cuantías de materias, por sí mismo traerá las mejoras, innovaciones, competencias y progreso del plan de estudios. A estas ideas se les llamó planificación y en ellas virtualmente estaba la fórmula para avanzar expeditamente. Follari (2010) señala a la sazón de esta tendencia:

Por una parte, la inicial cuestión sobre la formalización curricular hacia los años 60 del siglo XX, ponía el acento en la planificación. Se trataba de diseñar un buen plan de estudios, con programas de cursos que le fueran coherentes. Con esto pareció bastar; luego la tarea docente debía ceñirse a ese patrón preestablecido. Se supuso que la realidad se adecuaría a esas exigencias ideales. Pero la realidad es terca, y pronto se advirtió la insuficiencia de poner el acento sólo en el momento planificador (p.529).

Sin duda en principio la planeación es una forma pertinente de organizar el proyecto académico, sin embargo....

En Latinoamérica, las limitaciones de recursos y equipamiento, más las tradiciones culturales para las cuales el apego a determinismos no funciona, llevaron a que algunos teóricos se preguntaran - desde EEUU - por qué "estamos fracasando" en Latinoamérica (Follari, 2010, p. 529).

Este apego devoto al pensamiento y organización académica, que viene desde Norteamérica, siempre ha estado cargado de visos colonialistas y poco polemizado. Se establece como la fórmula y solución y como representante de lo moderno, de que es lo que nos permite actualizarnos y estar a la "par con el mundo". Objetarlo representa, en el contexto local, ser representante del atraso y el pasado, pocas veces se ve como una manera de decir que no funciona por x o y razones. En los estudios, hechos paradójicamente en Norteamérica, sobre nuestro contexto se señala curiosamente:

Además, en Latinoamérica, somos hijos de influencias centroeuropeas, de modo que al eficientismo crudo suele oponerse cierta incapacidad para lo pragmático, junto a una considerable tendencia de apego a lo teórico, a lo conceptual, que en el mundo sajón tiene menor cabida (Follari, 2010, p. 529).

Al programar políticas educativas (pedagógicas incluso), basadas en los "descubrimientos" exitosos foráneos, para aplicar en las universidades latinoamericanas, se parte impropiamente de contextos particulares que difieren en aspectos contextuales con los nuestros, por ejemplo, sobre la idea del currículo: 
La crítica apuntó sobre todo a mostrar que la cuestión curricular iba más allá de una buena planificación, que había que considerar las cuestiones institucionales, la perspectiva del docente, incluso el contexto social y político; el tema del currículum hace a toda la situación en que se inscribe el trabajo académico (Follari 2010. p. 529).

Esta tácita contradicción en el artículo mencionado, al hacer las observaciones críticas a lo foráneo pero recogiendo también sus observaciones, insistiendo en las necesidades contextuales, es precisamente el aspecto que se descuida en las políticas curriculares de nuestro entorno y sobre las cuales se hacen las sugerencias provenientes del mundo anglosajón que es referencia y ejemplo para usar en nuestro medio académico; es sobre la contradicción de cómo esta dicotomía es tomada en nuestro medio, y que muchas veces se establece como paradigma, sobre lo que insiste Follari. No se trata de negar a priori el pensamiento pedagógico y académico de otros lugares, el asunto es su relación con el contexto sociocultural.

En otras palabras, es de suma importancia dilucidar esta dualidad: reconocer que un plan por sí mismo, a pesar de su pertinencia, no resolvería las calidades de su aplicación si no tiene en cuenta el segundo aspecto, es decir la realidad contextual y el proyecto pedagógico.

Un plan de estudios adecuado es algo más que una planificación detallada: será también aquel que tenga en cuenta las efectivas posibilidades, que entusiasme a los alumnos, que interese a los futuros empleadores, que comprometa realmente a los docentes tanto en su conformación como en su aplicación concreta (Follari, 2010. p.529).

Ahora bien, es necesario reencontrar epistemológica y semánticamente la definición del currículum, de su uso, puesto que este concepto, casi eufemístico al tratar de dilucidar la significación de un plan académico de estudios, deambula sin rumbo y es usado caprichosamente.

Ya currículum dejó de ser sinónimo de plan de estudios, y aún de aplicación de éste, para ocuparse de toda la acción académica desarrollada en las instituciones educativas. Lo curricular salió del encapsulamiento, pero también se volvió coextensivo (equivalente) de la entera actividad institucional (Follari. 2010. p.529).

Esta suma de "soluciones" a través del plan curricular es un artificio que no permite entender donde se encuentran sus fronteras funcionales y convenientes, se transformó en comodín para supuestamente salvar la dignidad y pertinencia de un plan de estudios: 
Cada vez que hay crisis en los resultados del trabajo de la institución, se proponen cambios en el plan de estudios. Mágica posibilidad, el plan de estudio nos salvará. Poco importa que los docentes no se sientan interpretados en él, que tales docentes sean exactamente los mismos que estaban antes, pero con nuevas etiquetas en las materias asignadas, que el perfil profesional no se defina o que la coherencia entre cada curso y los demás (y la de cada uno con el conjunto) no se garantice. El plan de estudios aparece a menudo - aún hoy - como la receta universal, la posibilidad omnímoda, la nueva palanca habilitadora. "Dame un plan de estudio y moveré...” Por esto, es importante que no nos engañemos. Todos conocemos las posibilidades, pero también debemos reconocer los límites del recurso al cambio de plan. Estemos atentos con un ejercicio elemental de lógica: un buen plan de estudios es condición indispensable para lograr resultados de aprendizaje deseables. Sin duda. Pero no es condición suficiente: sólo eso no basta (Follari,2010. p. 529).

En nuestro medio universitario académico-artístico adolecemos abrumadoramente de estos galimatías al crear, justificar y defender un plan curricular para la materia e incrustarlo en el marco de los planteamientos generales de currículum de las universidades. No se entiende que en la práctica artística y en sus procesos formativos resulte profundamente subjetivo y vago valorar en cuantías de un plan "salvador" los resultados esperados en los procesos del intérprete, además de suponer que el hecho de que estén asentados en tales formulaciones, resuelve, de por sí, esos dilemas ${ }^{4}$.

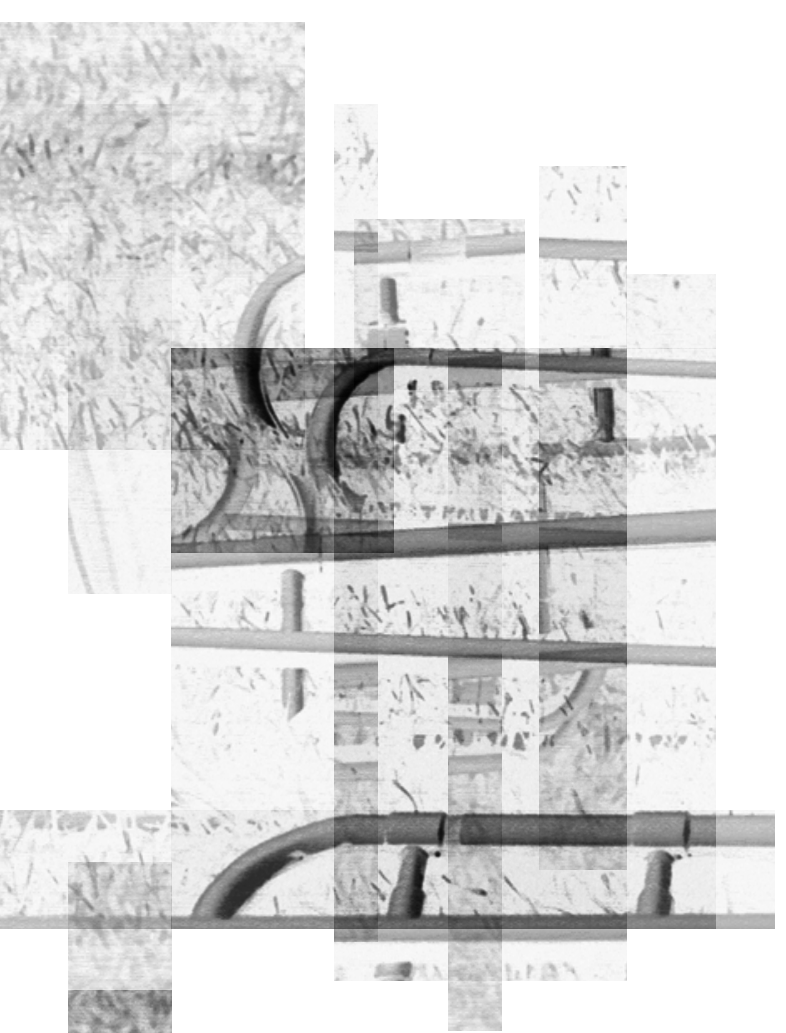

Al consultar con docentes de trombón, en el marco de esta investigación, estos dejaron muy en claro, dadas las condiciones y contextos propios, su incomprensión con la obligación a postulados curriculares de los cuales no han participado y que son siempre objeto de una resolución que viene de alguna parte jerárquica arriba de ellos (expuesto en las entrevistas del trabajo de campo de la tesis con los docentes de trombón).

Regresando a los créditos, tan importantes en reformas del currículo, encontramos un interesante escrito de Panquevo que, citando a la investigadora y pedagoga Lanora G. Lewis, plantea elementos de juicio y dudas al respecto de la distribución en créditos como mecanismo proficiente:

En el trabajo de Lanora G. Lewis, se establece que la cantidad de tiempo de clase (crédito por hora-clase) no constituye una medición del aprovechamiento del estudiante, por no atender factores como los arriba mencionados, y se recomienda que para indagar la verdadera relación que guardan 120 horas/ semestre con la instrucción, hay 
necesidad de: "establecer normas mínimas de graduación basadas en el aprovechamiento real del estudiante y no en la acumulación de créditos, con lo que se requiere ajustar el empleo de los créditos, para destacar logros y capacidades, más que la cantidad del tiempo pasado en el aula. (Lewis, 1972, citada en Panquevo, 1980, p. 2).

Este postulado es definitivamente válido en la práctica educativa artística. Los tiempos de "entrenamiento" funcional del instrumento implican trabajos diferentes con relación al nivel de desarrollo del educando; en las épocas de fortalecimiento y desarrollo del aparato para sonar (embocadura, respiración, lengua, etc.). Los lapsos de su práctica implican cantidades diferentes en la relación clase presencialno presencial. Como ha quedado bastante resaltado por los profesores de trombón, existen individualidades que obligan replanteamientos en cuanto el espacio de ejercitación personal y cumplimiento con el pensum de la clase según el grado de desarrollo técnico del estudiante. Por tanto, el pensum puede ser inscrito en tiempos de cada semestre, pero la actividad de entrenamiento debe ser amplia; esto quiere decir que la relación clase asistida con profesor y estudio personal deben guardar una relación suficientemente amplia, 30/70 en términos de porcentaje y a manera de ejemplo, pero no como un axioma. Además, no dejar de lado las competencias medibles relacionadas con aspectos técnicos y logros de competencias establecidas, y la subjetividad de la creación artística del intérprete, no medible, esto es objeto de valoración intrínseca, lo que de por sí establece una relación muy particular a la hora de "calcularse" en créditos. Puesto que no necesariamente, como una fórmula matemática, créditos es igual a conocimiento y competencia profesional.

Las políticas de créditos, que han reducido sus cantidades, han generado una superpoblación de contenidos que hacen caótico el plan curricular de la carrera. De tal manera que

un examen de las asignaturas de los diversos programas muestra una acreditación un poco alejada de los parámetros dados por la definición, especialmente con una fuerte tendencia hacia una baja acreditación, lo que conlleva una atomización de asignaturas en el programa que tiene como consecuencia una multiplicidad de cursos que es preciso atender cada semestre para poder reunir el número de créditos necesarios para ser alumno regular de la universidad (Panquevo, 1980, p. 6.)

La educación musical obliga un conocimiento intelectual muy amplio, artístico sin duda, pero también pedagógico y didáctico. Dice Regelski (1980):

...muchas facultades de música universitarias se resisten a la idea de que la enseñanza está basada en una disciplina firme, que está separada de la disciplina que se enseña, sucede a menudo que muchos maestros de música en perspectiva o con experiencia, son de alguna forma deficientes en las habilidades y aprendizajes que constituyen el arte de enseñar (p.20). 
Y este arte exige responsabilidad institucional y el reconocimiento de la especificidad de la carrera musical en sus características. El sistema de educación primaria y secundaria pública, lamentablemente, no cumple con estas necesidades, y cada vez lo poco que se implementa se esfuma. La universidad debe entender la importancia estructural de esta área de conocimiento y fortalecerla desde la infancia.

No establecer, para la implementación del pregrado en Trombón, las bases de su aprehensión y desarrollo desde la niñez, transforma en absurdo pretender, a través de escuetos contenidos de un pensum (y su confusión en el currículo), resolver la validez y calidad de un programa en la carrera. La política de créditos, que fue en sí una buena idea, debe tener la posibilidad de revivir y replantearse al aplicarla para la carrera específica de instrumentista y en la generalidad del plan curricular.

Instamos, en la particularidad del pregrado en Trombón, no debe ser un curso en instrumento que está lleno de contenidos propios a otras especialidades de la música y de otras de carácter general formativo. Debe ser competente como cualquier otro pregrado profesional, y no la especie de curso de "de todo un poco" que es hoy; además, insistimos con obstinación, debe existir una seria, profunda y profesional reforma a los programas infantiles, desde su inclusión en el currículo de la unidad académica, hasta la calidad y competencia de los docentes que abordan esta tarea. Señala muy pertinentemente García Gallego y García Quiroz (2011) con relación a este pensamiento:
Cuando se habla de educación artística, se encuentran concurrencias a la manera de ser abordado; son diversos los autores y publicaciones que han tratado el tema de la educación artística infantil, no obstante, es reiterativo como en algunas instituciones educativas no se ha dimensionado su esencia formativa (p. 12).

Sin dilucidar la importancia de esa "esencia formativa" para trabajar y preparar a los niños, a través de un camino pedagógico competente de educación artística en música-instrumento, las vías para el perfeccionamiento de la carrera, y su consolidación internacional, seguirá siendo aleatorio y fortuito.

El crédito académico se asocia al conflicto con la diversidad programática en el sistema nacional de educación instrumental. Allí topamos que es decididamente diferente entre cada institución, no existe un plan nacional sobre el programa de instrumento trombón. Las instituciones mantienen, en otras áreas de conocimiento, intercambio

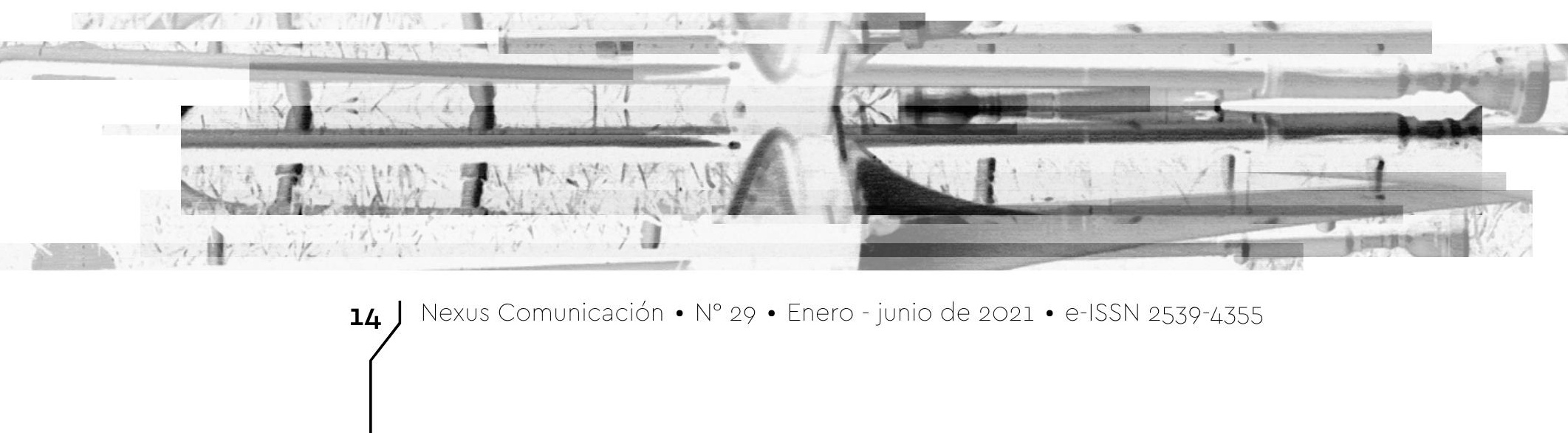


bastante loable y aplicado en ese sentido, sin embargo, en los programas de trombón esto es aún irrelevante. Los contenidos por grados en las clases de trombón tienen similitudes, pero no establecen parámetros que los regulen y sustenten.

El uso de textos, métodos de trombón en las universidades públicas con pregrado de instrumento tiene analogías en autores y algunos aspectos de contenido, pero en ninguna parte fue clara la respuesta a preguntas como las siguientes: ¿Por qué, en qué tiempo del desarrollo? ¿Cómo regular sus contenidos? o sea hasta ¿Dónde se aplica en cada semestre o nivel del curso? ¿Cuál es la interrelación con otros textos? ¿Cuál es la sustentación pedagógica? Dada la desigualdad formativa para acceder al curso, caracterizada por la diversidad contextual y académica, es complejo entender ¿Cómo se usa un mismo texto en personas sin ninguna formación y en los que ya traen algunas bases? Ciertamente en la cabeza de los profesores, y por lo escuchado en las entrevistas, hay una idea, solo que ella difiere en algunos casos completamente. A diferencia de otras carreras no se encuentra mucho material pedagógico de apoyo, como suele ya suceder en EEUU y Europa.

Los fundamentos expuestos sobre su aplicación están basados en la tradición y la experiencia, entendida esta última más como lo empírico que lo conceptual. Sin embargo, no puede, y no debe, un contenido curricular partir del anacrónico prejuicio "romántico" de la individualidad y cognición del artista como fundamento y argumento de la idoneidad del curso. Con todo el respeto y consideración que tenga, indudablemente, el artista en cuestión, un sistema nacional de enseñanza no puede ser subyugado al espíritu subjetivo que basa, en la experiencia del artista, y en perspectiva docente, el cimiento de su competencia.

Los programas de instrumento deben alcanzar una aprobación conceptual y pedagógica general. No se trata de usar los mismos textos y autores, pero sí de definir contenidos y competencias ajustadas a un plan nacional en el cual, indiferentemente del autor y el texto, estas competencias tengan equidad programática y sirvan de referente en cualquier universidad pública; en otras palabras deben tener un paralelo de contenidos, evaluaciones y resultados que, en medio de la relatividad contextual y de condiciones del alumno, le genere la confianza de ir por un camino delineado en cuanto sus objetivos y resultados.

El lector podrá encontrar en lo dicho contradicciones con lo señalado en relación a los créditos, la individualidad del alumno, el talento, etcétera. Rehagamos, no se trata de un plan curricular VIP o simple y formalmente unificado, sin duda no se trataría de eso. Es necesario trabajar en un frente común para que los textos tengan similitudes programáticas y parámetros que permitan establecer líneas de competencia medibles en un mínimo exigido por contenidos y, sobre todo, competencias adquiridas. Un curso profesional debe seguir un derrotero programático en el cual los objetivos generales y particulares no sean un formalismo burocrático de cumplimiento alegórico con la institución en la que se trabaja. Aquí 
la llamada flexibilidad, intercambio universitario, transversalidad, etc. deben dejar de ser letra altiva en documentos doctos y transformarse en honestidad prometida sobre la formación idónea del educando y evitar que este al graduarse sienta

que no los prepararon exactamente para aquello en que deberán trabajar; a veces, simplemente no pueden especificar cuáles serían sus funciones en la sociedad. Se los formó pensando en los contenidos científicos de su disciplina, pero no en el ejercicio profesional de su rol. Es cierto que no podemos abandonar la reflexión académica en aras de un pragmatismo miope, como el que predomina en estos tiempos: sin duda es imprescindible sostener la coherencia científica, y el valor de la teoría y de la discusión conceptual. Pero no olvidemos direccionarnos también al rol profesional, si no queremos quedar disociados de la demanda social y colaborar así a la futura desocupación de los profesionales, ya favorecida por razones económicas estructurales (Follari, 2010, p. 532).

Significa que la flexibilidad y la individualidad deben tener molduras programáticas, de lo contrario sería la continuación de la anarquía que vivimos hoy y del desfase curricular; cuidado, no confundir con las habilidades del talento y las características individuales de los artistas. Son dos aspectos mutuos pero diferentes en su esencia: el artista es una individualidad, las competencias profesionales una generalidad, de allí la palabra profesional, y la titulación de una profesión.

Los libros didácticos, llamados métodos de autores extranjeros, y todos aquellos que tienen similitudes deben encontrar un espacio y organización conceptual, metodológica y de uso pedagógico en los tiempos de la carrera. La individualidad permitirá ir más o menos rápido, pero estos contenidos deben tener razón de ser en cada paso de la formación del estudiante. No deben usarse porque sí, deben ser justificados didácticamente sus contenidos, los tiempos y lugares a llegar por semestre, deben existir puntos de partida y llegada. No es educación medieval, está inscrita en sistemas públicos de educación superior y debe ser congruente con ello. Esta es una preocupación restringida y vigente sobre los docentes artistas en los pregrados universitarios de la universidad pública, dice Regeleski (1980), sobre la individualidad del educador, sobre el soporte basado en la experiencia

Aquellos que formalmente desean ser maestros de música- educadores en la música-, deben de ir un paso más allá de sus capacidades musicales, para dominar la ciencia de la enseñanza de la música. Aunque siempre habrá margen para las decisiones puramente humanas y para funcionar en cualquier aspecto de la enseñanza, se pueden dejar pocas decisiones respecto a la naturaleza específica de la instrucción, únicamente al capricho de los maestros individuales (p. 21).

Esto quiere decir que es necesario un estudio profundo, sistemático y funcional del uso y aplicación de los métodos de trombón. No se puede, ni debe, entregarle al alumno unos libros de métodos, decirle: "estudie del número tal al tal, y ya". 
Eso es poco profesional, didáctico y práctico, no contribuye efectivamente a la formación del estudiante. Deben establecerse con rigor ¿cuáles y porqués? de los contenidos, definir los resultados básicos esperados, de cada curso por semestre, en cuanto competencias y aprehensiones técnicas. Las temáticas deben parecerse, los textos no necesariamente. Y definitivamente debe crearse una sustentación teórica de los resultados esperados, de los contenidos a estudiar. Es necesario orientar al alumno desde el principio a investigar, a buscar la pertinencia de la estrategia y la táctica del docente, a confrontar y contestar lo que se le ordena estudiar. Se trata de convencer no de imputar. La educación musical es científica por antonomasia en sus competencias técnicas y emocional por defecto en sus características expresivas. Los programas deben parecer una ruta, un mapa válido en la geografía educativa universitaria. Los métodos europeos y norteamericanos, con su sólida tradición y validez, deben inmiscuirse en nuestra realidad, deben ser estudiados, definidos programáticamente, $\mathrm{y}$, sin duda, es hora de generar metodologías resultado de nuestra actividad nacional educadora del trombón, métodos colombianos que impliquen esa vasta cognición universal, pero también la nuestra. No es "patriotismo" es pensar en el desarrollo lógico de años de trabajo educativo trombonistico en Colombia. Hoy se escribe mucho texto hiperbólico sobre temáticas educativas, pedagógicas, psicológicas de la enseñanza, etcétera., pero poco es su uso en la realidad y praxis educativa nacional, necesitamos hacerlo y aplicarlo. Es decir, concluimos que es fundamental darle relevancia y aplicación a los aspectos ontológicos, cognitivos y sociológicos como fuente de partida en el mejoramiento y equitatividad de las aplicaciones metodológicas en el pregrado de Trombón. Sin duda debe cambiar el ethos de la clase, este debe ser un mundo transformador, estimulante, creativo y crítico. Es necesario un currículo unificado nacional, no un rasero de contenidos y sí una comprensión pedagógica de competencias que resulte de un profundo intercambio pedagógico entre los entes universitarios públicos. 


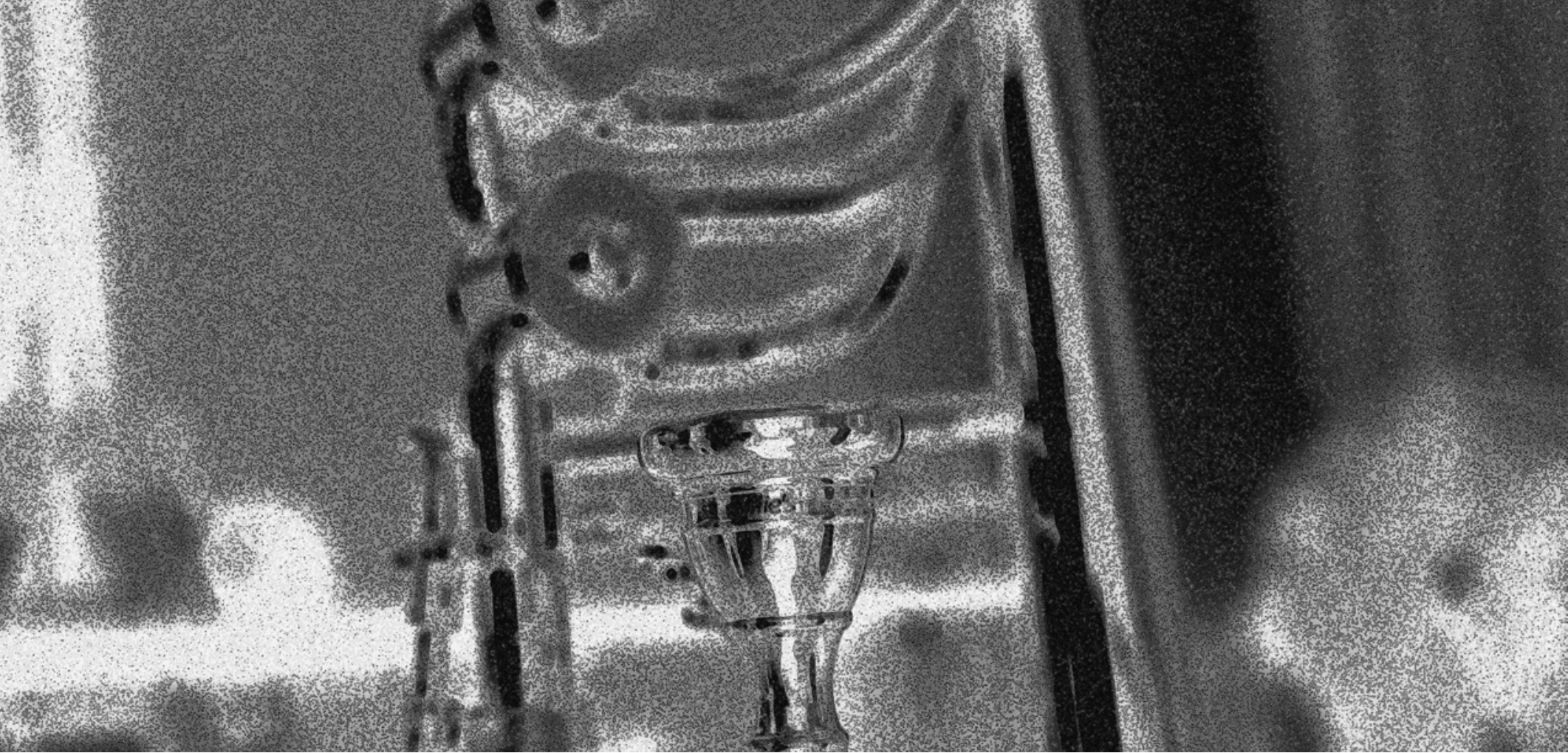

\section{Referencias}

Bourdieu, P. (2005). Campos de conocimiento: teoría social, educación y cultura. Facultad de Humanidades.

Cárdenas, R. (2012). Evaluación de las Titulaciones de Licenciatura en Música en Colombia: análisis exploratorio y opinión del profesorado implicado [tesis de doctorado, Universidad de Granada]. Repositorio Institucional Universidad de Granada. https: / / digibug.ugr.es/handle/10481/26388

Follari, R. (2010). Curriculum y doble lógica de inserción: lo universitario y las prácticas profesionales. Cadernos de Pesquisa, 40(140), 529-546. https://doi.org/10.1590/S010015742010000200011

García, A. \& García, C. (2011). La educación artística: un estado del arte para nuevos horizontes curriculares en la institución educativa [tesis de maestría, Universidad Tecnológica de Pereira]. Repositorio Institucional UTP. https://1 library.co/document/yng0p1pz-educacionartistica-horizontes-curriculares-institucion-educativa-ciudad-pereira.html

Constitución Política de Colombia (1991). Artículo 67.

Ministerio de Educación Nacional de la República de Colombia (2010). Orientaciones Pedagógicas para la Educación Artística en la Básica y Media. Ministerio de Educación Nacional de la República de Colombia.

Presidencia de la República de Colombia (2015, 26 de mayo). Decreto 1075. Por medio del cual se expide el Decreto Único Reglamentario del Sector Educación. Ministerio de Educación Nacional de la República de Colombia. https://www.mineducacion.gov.co/1759/w3-article-351080. html?_noredirect $=1$

Panquevo, J. (1980). Algunas consideraciones sobre los "créditos académicos" o "unidades de labor académica”. Revista Colombiana de Educación,(6).https: / / doi.org/10.17227/01203916.5046

Regelski, T. A. (1980). Principios y problemas de la educación musical. Diana.

Revista Semana (1990, 28 de octubre). El síndrome del niño rico. Revista Semana. https://www.semana.com/vida-moderna/articulo/el-sindrome-de-nio-rico/14059-3/

Ulloa, A. (1989). La salsa en Cali: cultura urbana, música y medios de comunicación. Boletín Socioeconómico, (19), 141-154. https://bibliotecadigital.univalle.edu.co/ handle/10893/5481

Universidad del Valle (2015, 25 de septiembre). Acuerdo 025. Por el cual se actualiza la Política Curricular y el Proyecto Formativo de la Universidad del Valle. http://proxse16.univalle.edu.

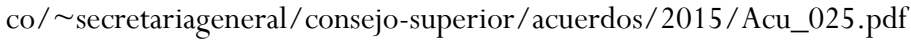




\section{Notas}

${ }^{1}$ Profesor de la Escuela de Música de la Universidad del Valle (Cali, Colombia). Doctor en Historia de las Artes, Universidad de Granada. Master of Fine Arts, Conservatorio Rimsky Korsakov, San Petersburgo (Rusia). Maestro en Música con énfasis en Trombón, Conservatorio Antonio María Valencia (Cali, Colombia). Director de la Orquesta Sinfónica de Estudiantes de la Escuela de Música y de la Banda Sinfónica de la Universidad del Valle.

${ }^{2}$ La metodología de investigación incluyó lo siguiente:

a. Revisión de fuentes: a partir de la recolección, compra de textos, y consulta con los profesores encargados de las cátedras de trombón de las universidades consultadas sobre los libros de método y calentamientos para trombón usados en nuestras instituciones (WarmUp, anglicismo que se usa en el medio académico trombonístico), se procedió a compilarlos por escuelas, y a organizarlos en lo posible cronológicamente para revisar sus contenidos programáticos y propuestas metodológicas.

Se llevaron a cabo diversos encuentros que permitieron la elaboración de cuestionarios, encuestas, entrevistas y foros académicos. También se realizaron visitas a todas las universidades citadas para verificar en fuentes primarias sobre decretos y resoluciones creadoras de los programas de trombón para pregrado. Allí mismo se realizaron video entrevistas con estudiantes y profesores sobre los asuntos pertinentes a las cuestiones de esta investigación.

Teniendo este material he procesado sus informaciones en puntos concordantes y disímiles, y he creado una base de datos con estos métodos. Así mismo he consultado en todas las fuentes posibles y de mi alcance, bibliotecas locales, bases de datos e Internet, para recuperar informaciones pertinentes al tema.

b. Revisión crítica de las metodologías: con el propósito de diseñar unas conclusiones que aborden de manera crítica, e incluyan elementos conceptuales, las pertinencias y mejoras competentes para la aplicación de estas metodologías al servicio del desarrollo y crecimiento académico de nuestros alumnos y programas.

${ }^{3}$ Subrayado por el autor.

${ }^{4}$ Un ejemplo gráfico, para reflejar esa manía "innovadora-salvadora” de cambiar el currículo cada que este no produce los resultados esperados y que como respuesta a ello propone cambios del plan curricular para "mejorar", y que nos podría ilustrar sobre la nulidad de estas innovaciones, lo encontramos en el paisaje arquitectónico de nuestras ciudades, en las cuales se destruyó la memoria arquitectónica, el legado de belleza y arte urbanístico de otrora, en nombre de la "modernidad y el cambio", y hoy nuestras ciudades son caóticas, sucias, sin coexistencia estética, ecológica, y con el entorno abruptas, sin contexto. Los “cambios” no bastaron ni generaron solución al problema urbano en sí. 